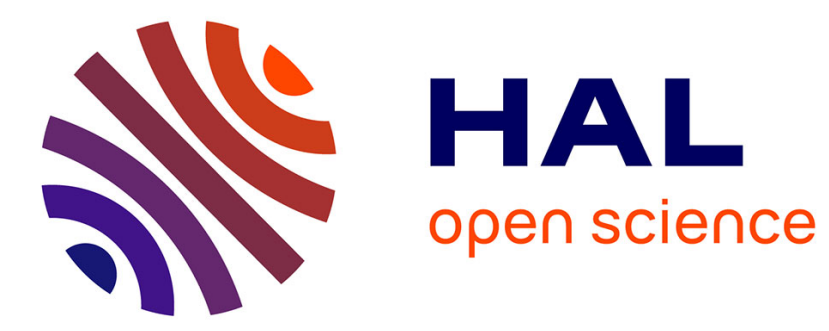

\title{
Population balance approach for the modelling of enzymatic hydrolysis of cellulose
}

Noureddine Lebaz, Arnaud Cockx, Mathieu Sperandio, Jérôme Morchain

\section{To cite this version:}

Noureddine Lebaz, Arnaud Cockx, Mathieu Sperandio, Jérôme Morchain. Population balance approach for the modelling of enzymatic hydrolysis of cellulose. Canadian Journal of Chemical Engineering, 2015, 93 (2), pp.276 - 284. 10.1002/cjce.22088 . hal-01876382

\section{HAL Id: hal-01876382 \\ https://hal.science/hal-01876382}

Submitted on 16 Nov 2020

HAL is a multi-disciplinary open access archive for the deposit and dissemination of scientific research documents, whether they are published or not. The documents may come from teaching and research institutions in France or abroad, or from public or private research centers.
L'archive ouverte pluridisciplinaire HAL, est destinée au dépôt et à la diffusion de documents scientifiques de niveau recherche, publiés ou non, émanant des établissements d'enseignement et de recherche français ou étrangers, des laboratoires publics ou privés. 


\title{
POPULATION BALANCE APPROACH FOR THE MODELING OF ENZYMATIC HYDROLYSIS OF CELLULOSE
}

\author{
Noureddine Lebaz, ${ }^{1,2,3,4}$ Arnaud Cockx, ${ }^{2,3,4}$ Mathieu Spérandio, ${ }^{2,3,4}$ and Jérôme Morchain ${ }^{2,3,4 *}$ \\ 1. Toulouse White Biotechnology, 3 rue des satellites, 31400 Toulouse, France \\ 2. Université de Toulouse; INSA, UPS, INP; LISBP, 135 Avenue de Rangueil, F-31077, Toulouse, France \\ 3. INRA, UMR792 Ingénierie des Systèmes Biologiques et des Procédés, F-31400, Toulouse, France \\ 4. CNRS, UMR5504, F-31400, Toulouse, France
}

\begin{abstract}
In this numerical work, a population balance based model is proposed in order to describe the cellulose particles size evolution during the enzymatic hydrolysis. Two kinds of actions are considered: endoglucanase activity that cleaves randomly $\beta$-1,4-glycosidic linkages of cellulose and exoglucanase activity which reduces the particles size with chain-end-cleaving producing cellobiose (a dimer of two glucoses linked by a $\beta$-1,4-glycosidic bond). A discretization method with a fixed pivot technique is used for the endoglucanase action and a moving pivot technique for exoglucanase attack. The numerical resolution is then validated by analytical solutions available in literature. Afterwards, the combination of the two actions is investigated for different enzyme ratios in order to reproduce the endo-exo synergism numerically. Since the biodegradation of cellulose releases D-glucose as a final product due to $\beta$-glucosidase which hydrolyzes cellobiose into two molecules of glucose, numerical kinetic model predicting the fractional conversion of cellulose is derived from the population balance developed model. The enzymes activity is strongly affected by the accumulation of the end-products (cellobiose and glucose) during the hydrolysis, the inhibition effect is thereby incorporated in the model. The numerical model prediction is compared to experimental data in the case of combined activity and shows a promising approach for the modeling of cellulose-cellulase systems.
\end{abstract}

\section{Introduction}

The use of lignocellulosic materials, known as the largest renewable carbohydrate source on earth, ${ }^{[1]}$ for biofuels and chemicals production has been widely studied since the global supply of fossil fuels is limited. ${ }^{[2-4]}$

The focus has been on the bioconversion of biomass by means of hydrolytic enzymes capable of degrading these insoluble materials to soluble and fermentable sugars. However, both the enzyme systems and the understanding of cell wall structure are still subject to improvement. ${ }^{[5]}$ Indeed, the hydrolysis reaction, considered as one of the main limiting steps of the bioconversion process, ${ }^{[6]}$ has a multidimensional mechanism. It depends on the chemical and structural substrate features (chemical composition especially lignin content, accessibility, reactive area .... $)^{[7,8]}$ as well as on the biocatalyst (nature and enzymatic cocktail composition) ${ }^{[9,10]}$ and on the operating conditions (temperature, $\mathrm{pH}$ and mixing). ${ }^{[11,12]}$ In addition to that, one has to consider complex substrate-biocatalyst interactions which remain to be elucidated. ${ }^{[13]}$ This complexity makes the kinetic modeling of such systems delicate and

\footnotetext{
*Author to whom correspondence may be addressed: jerome.morchain@insa-toulouse.fr
} 
the experimental determination of the role of each parameter on the whole reaction unobvious.

Nevertheless, several kinetic models based on different assumptions have been developed for the enzymatic hydrolysis of pure cellulose and lignocellulosic materials, reviewed by Bansal et al. ${ }^{[14]}$ Michaelis-Menten based models, developed initially for homogenous reaction (such as the action of $\beta$-glucosidases on soluble cellobiose) have been used by many authors to describe the whole hydrolysis process. ${ }^{[15,16]}$ Since hydrolysis is a heterogeneous reaction, the overall rate depends on the amount of adsorbed cellulases. Adsorption models based generally on Langmuir adsorption isotherm are, thereby, used for fitting experimental data. ${ }^{[17,18]}$ In these models it is assumed that the limiting step is related to the enzyme adsorption. Irreversible adsorption on lignin has been reported for lignocellulosic materials ${ }^{[19,20]}$ as well as nonreactive adsorption on crystalline cellulose. ${ }^{[21,22]}$ It is important to observe that MichaelisMenten and adsorption models share the same mathematical formalism. The parameters have different meaning but they are only apparent and global constants. For example, the fact that the specific area changes during the reaction because of the substrate liquefaction cannot be taken into account by these models. ${ }^{[23]}$

More comprehensive models have been proposed integrating the rate limiting factors such as the synergistic interaction of cellulases, the activity inhibition by products accumulation and the enzyme accessibility to the hydrolysable fractions. ${ }^{[18,24]}$ But they are based on the same approach as those described before. Movagarnejad et al. combined Langmuir isotherm concept and shrinking particle theory ${ }^{[25]}$ assuming that the particles are cylindrical and preserve their shape during the shrinkage process with an invariant diameter to height ratio. The weakness of such models lies in their inability to integrate heterogeneous particles size distribution with different shapes.

In the light of these considerations, population balance modeling can be an alternative for developing mechanistic models for systems as complex as lignocellulosic materials hydrolysis. In fact, population balance equations, used for diverse applications, draw strength from their ability to describe dynamic systems evolution taking into consideration particular level processes (breakage, aggregation ...). Except Hosseini and Shah, ${ }^{[26,27]}$ no literature could be found using this approach for enzymatic lignocellulosic materials hydrolysis. In their discrete models, Hosseini et al. assimilated the cellulose hydrolysis to a linear chain depolymerisation. But for cellulose hydrolysis by endoglucanase, the discretization scans all 
possible polymer lengths leading to an onerous resolution since the number of equations is proportional to the maximum initial chain length.

In this paper, we develop models for cellulose particles size evolution during their hydrolysis by endoglucanase and exoglucanase enzymes. For the resolution of the population balance equations, we use the discretization method developed by Kumar and Ramkrishna ${ }^{[29-31]}$ with a fixed pivot technique for the endoglucanase activity and a moving pivot technique for the exoglucanase attack. Once these two models are validated, a combined model for both activities is proposed and solved using the moving pivot technique. This model predicts the particles size distribution during hydrolysis as well the conversion yield of the substrate. The originality of the work proposed here is to incorporate enzyme activity mechanisms into the robust framework of population balance modeling. Finally, the extension of the developed model in order to take into account the end-products inhibition effect is explored.

\section{Model framework and numerical method}

Population balance general equations describe particulate systems behavior when submitted to a dynamic evolution of their physical or chemical properties. In our case, we consider pure cellulose particles assimilated to polymer chains with a given initial size distribution. Enzymatic hydrolysis of this substrate leads to a progressive decrease in the chains length due to breakage and chain-end-cleaving processes. As a first step, each enzymatic activity is treated separately and the associated numerical method is presented. Then, the full model combining the two activities is described.

For a continuous and homogeneous system, the evolution of the particles size distribution undergoing binary break-up and constant growth is described by the kinetic equation: ${ }^{[30]}$

$$
\frac{\partial n(x)}{\partial t}=-\frac{\partial[G(x) n(x)]}{\partial x}-n(x) \Gamma(x)+\int_{x}^{\infty} \beta\left(x, x^{\prime}\right) \Gamma\left(x^{\prime}\right) n\left(x^{\prime}\right) d x^{\prime}
$$

The first term on the right hand side corresponds to the net accumulation related to the growth of particles which is a kinetic phenomenon. The second term represents the loss (death) of particles with a size $x$ due to their break-up (with a frequency $\Gamma(x)$ ). The last term describes the production of new particles with a size $x$ (birth) due to the breakage of larger particles. The birth and death terms are discrete events. The last integral term represents the main issue in solving the equation (1). Except in some particular cases for which analytical solutions exist, population balance equations resolution is carried out by numerical techniques. Here, 
we chose the discretization method (method of classes) developed by Kumar and Ramkrishna. ${ }^{[28]}$

\section{Discretization method}

The discretization method consists of dividing the entire property $\xi$ range (size, volume, specific area ...) into a finite number of intervals, noted $M$. All particles for which the property $\xi$ lies in the interval $\left[\xi_{i} ; \xi_{i}+d \xi_{i}\right]$ are attributed to the $i^{\text {th }}$ interval represented by its center $x_{i}$ called pivot. Thus, the initial problem is transformed into a set of $M$ equations, regarding the number of particles, $N_{i}(t)$, in the $\mathrm{i}^{\text {th }}$ interval. The integral term in each equation is approximated numerically using the same discretization.

\section{Modeling the endoglucanase activity}

The hydrolysis due to endoglucanases solely is assimilated to a pure breakage process ( $G=0$ in equation (1)). Endoglucanases are known for internally cleaving cellulose chains (figure 1). Their random actions on $\beta$-1,4-glycosidic bonds lead to binary breakages without any position preference but as admitted by Hosseini and Shah, ${ }^{[26]}$ the breakup frequency is a function of chain length since longer chains are more susceptible to be attacked. Thus, as a first approach, we consider the breakage frequency $\Gamma$ proportional to cellulose chains length.

As the polymers breakage is a discrete process, it has a probabilistic foundation. Considering a cellulose chain with an initial length $x^{\prime}$, we look for the probability $\beta\left(x, x^{\prime}\right)$ to form a new chain with a length $x$ after the breakup of the chain $x$ '. Since the breakage is binary, there are only two possible locations along the initial chain length $x$ ' on which the enzymes can act in order to form the chain $x$ (figure 1). Thus, the probability expression is $2 / x^{\text {, }}$. 31$]^{\text {. }}$

During the population particles evolution, new particles are formed by breakup. The size of the newly formed particle may not correspond to an existing pivot. So this particle has to be affected to the neighboring pivots. Considering a new particle with a size $\xi$ in the range $\left[x_{i}\right.$, $\left.x_{i+1}\right]$, one has to calculate the fractions $a\left(\xi, x_{i}\right)$ and $b\left(\xi, x_{i+1}\right)$ that will be affected to the pivots $x_{i}$ and $x_{i+1}$. Following the approach proposed by Kumar and Ramkrishna, we chose to preserve the particle number and mass, noted $f_{1}$ and $f_{2}$, which lead to equations (2) and (3). ${ }^{[28]}$

$$
\begin{aligned}
& a\left(\xi, x_{i}\right) f_{1}\left(x_{i}\right)+b\left(\xi, x_{i+1}\right) f_{1}\left(x_{i+1}\right)=f_{1}(\xi) \\
& a\left(\xi, x_{i}\right) f_{2}\left(x_{i}\right)+b\left(\xi, x_{i+1}\right) f_{2}\left(x_{i+1}\right)=f_{2}(\xi)
\end{aligned}
$$


The breakage process is defined as a continuous event over a given cellulosic chain length. It could be thought that this is in contradiction with the fact that breakup actually occur at the glycosidic linkages. In fact, the new formed chains appearing in the system are distributed on two adjacent pivots, as described previously. This solves the apparent contradiction since the pivots correspond to polymer lengths which are physically possible.

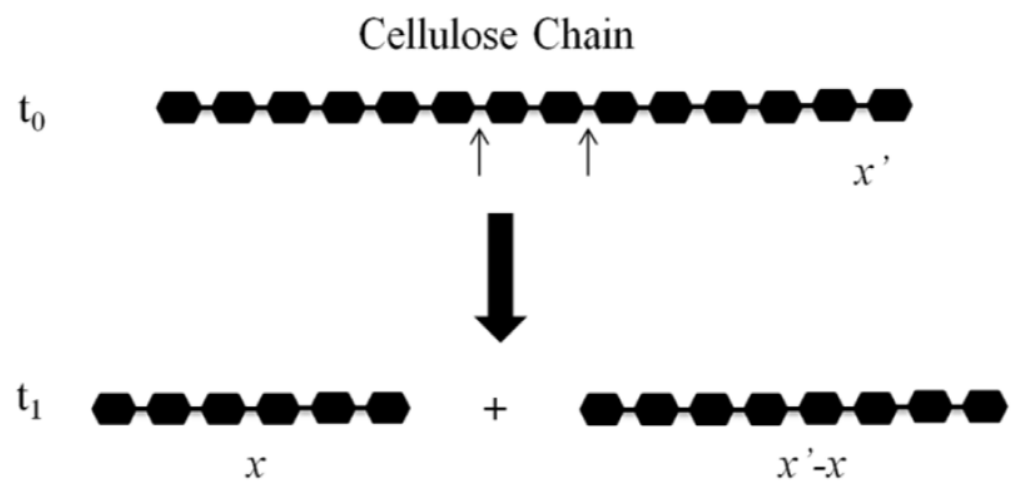

Figure 1: Schematic representation of the endoglucanases activity. Random scission with a given nature (binary in this scheme) and breakage frequency $\Gamma$. The arrows show the two possible locations to have a particle of length $x$ after the breakage of $x$ '.

After several mathematical transformations, Kumar and Ramkrishna establish the discrete equation for the pure breakup process as the linear sum of the birth and death particles terms given below: ${ }^{[28]}$

$$
\frac{d N_{i}(t)}{d t}=-\Gamma_{i} N_{i}(t)+\sum_{k=i}^{M} n_{i k} \Gamma_{k} N_{k}(t)
$$

The left hand side term of the equation (4) corresponds to the variation of the total number of the particles in the $i^{\text {th }}$ interval $(1 \leq i \leq M)$ per unit time. This variation is the consequence of two phenomena appearing on the right hand side of the equation. The first term refer to the particles in the $i^{\text {th }}$ interval disappearing at the frequency $\Gamma_{i}$ because of hydrolysis. It corresponds to the number of the particles of length $x_{i}$ undergoing breakage process per unit time, i.e. the second term on the right hand side of equation (1). The second phenomenon is related to the particles of length $x_{k} \geq x_{i}$ which may give, after breakage, at least one particle in the $i^{\text {th }}$ interval. The number of the new particles appearing in the $i^{\text {th }}$ interval per unit time is given by the second term of the equation (4) corresponding to the discretization of the integral term of the equation (1). For further details regarding the calculation of $n_{i k}$, please refer to the original work cited. It is worth noting that these $n_{i k}$ values can be calculated a priori, i.e. before starting the resolution of the set of equations (4). Indeed, the $n_{i k}$ values depend only on the properties $f_{1}$ and $f_{2}$ and the value of the pivots. 
The numerical resolution of the discretized population balance equation was performed using the fixed pivot technique and was validated by analytical solutions available in literature. Ziff and McGrady proposed analytical solutions in the cases where the breakage frequency is $(\Gamma=1)$ or $\left(\Gamma=x^{\alpha}\right) .{ }^{[31]}$ Once the model is validated, other breakage frequency mathematical expressions can be easily implemented.

\section{Modeling the exoglucanase activity}

The second major hydrolytic enzyme is exoglucanase, called also cellobiohydrolase. It acts on the ends of cellulose chains and releases cellobiose (figure 2). This action causes a progressive decrease of the cellulose chains length. Unlike endoglucanases requiring probabilities for predicting daughter particles length, exoglucanase activity leads invariably to a chain with a degree of polymerization decreased by two after each attack. An equal reactivity, irrespective of the chain length, is assumed.

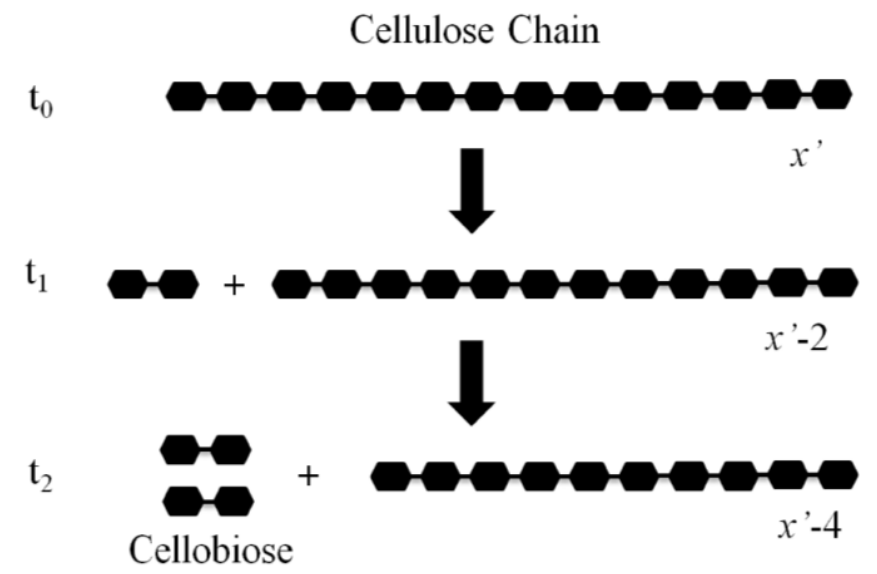

Figure 2: Schematic representation of the exoglucanases activity. Each attack produces one cellobiose and reduces the length of the initial chain by two monomers.

We propose to model this hydrolytic activity as a pure growth ${ }^{[30]}$ with a negative rate defined as the rate of decrease of the chains length $(G(x)=d x / d t)$. The hydrolysis by exoglucanases is thus described as a continuous dissolution of the cellulose polymers. The equation to solve is obtained by setting $\Gamma=0$ in equation (1). In that case, the moving pivot technique ${ }^{[29]}$ is more appropriate for resolving the discretized population balance equation. The gradual decrease of the cellulose chains length can be related to the pivot moving rate, thus the technique reproduces indirectly the enzymatic hydrolysis rate. Generally, the moving pivot rate is either 
constant $\left(G(x)=\sigma_{0}\right)$ or depending on the particles size $\left(G(x)=\sigma_{0} x\right)$. In the two cases, analytical solutions exist ${ }^{[30]}$ and are used in this work to validate the numerical results.

Compared with similar growth systems available in literature, ${ }^{[33-34]}$ it should be noted that in our case, in view of negative moving rate (rate of the chains dissolution by the exoglucanase activity), we must avoid switching to negative pivots which is nonsense. Note that the first pivot corresponds to a chain length of 2 monomers of glucose (cellobiose). Considering this, we set the first pivot equal to the smallest chain length in the system (cellobiose). This pivot is not moving. The other pivots move to the left at a constant rate, this means that all the cellulose chains (except cellobiose) are attacked at a same frequency. The first pivot being fixed, knowing the initial chain lengths and the moving rate, the time necessary for the second pivot to reach the first one is calculated as:

$$
\tau=\frac{x_{2}(t)-x_{1}}{G(x)}
$$

The PBE is integrated over this time interval. At the end of the time interval, the second pivot is eliminated and its final number density or total particles number is assigned to the first pivot. The procedure is repeated until the final simulation time is reached.

Since the pivots level is a chain length, their moving to smaller chain lengths induces weight loss corresponding to the produced cellobiose which can be easily evaluated. The total mass of the polymers is conserved through the process. The first moment of the distribution multiplied by the molar mass of the monomer (glucose in the present case) corresponds to the total mass of all chains. Given the initial mass, $m_{T}$, is known and that the PBM leads to the chain length distribution at any time, the difference between the initial mass and the mass of all chains longer than 2 corresponds to the mass, $m_{c}$, that was transformed into cellobiose. Then it comes that

$$
\frac{m_{c}(t)}{m_{T}}=\frac{m_{T}-\frac{M_{G l u}}{\mathscr{N}} \int_{x_{2}}^{\infty} x N(x, t) d x}{m_{T}}
$$

Where the integral term corresponds to the total mass of the chains larger than cellobiose. Being capable of assessing the amount of the cellobiose produced during the process by means of the equation (6), one can deduce at any given time the fraction of converted substrate.

\section{Combined model and synergistic interaction of cellulases}


The discretized population balance equation (1) for a combined endoglucanase-exoglucanase activity is resolved with the moving pivot technique. This technique is chosen because it is judicious to treat the pure growth (exoglucanase activity) part of the equation (1). However since the pivot are now moving, the $n_{i k}$ values involved in the breakage part of the equation are no longer constant and their values have to be updated throughout the solution procedure as proposed by Kumar and Ramkrishna. ${ }^{[30]}$

The resolution of the discretized population balance equation in the different considered cases was performed with Matlab® software.

\section{Results and discussion}

\section{Endoglucanase activity}

As discussed previously, the discretized population balance equation in the case of the endoglucanase activity is resolved with a fixed pivot technique. The initial distribution used in order to validate the numerical approach is monodisperse (all the chains have the same initial length). The breakage frequency depends on the particles size $(\Gamma=x)$ and the analytical solution has been discussed by Ziff and McGrady. ${ }^{[31]}$

The Figure 3a represents a comparison between analytical and numerical number distibutions during the hydrolysis reaction by endoglucanase. The Figure $3 b$ represents the effectiveness of the breakage in terms of new particles formed in the system during the reaction. The numerical results are very close to the analytical solutions in the two cases, the error is insignificant at this level. The numerical approach is, thereby, validated. 


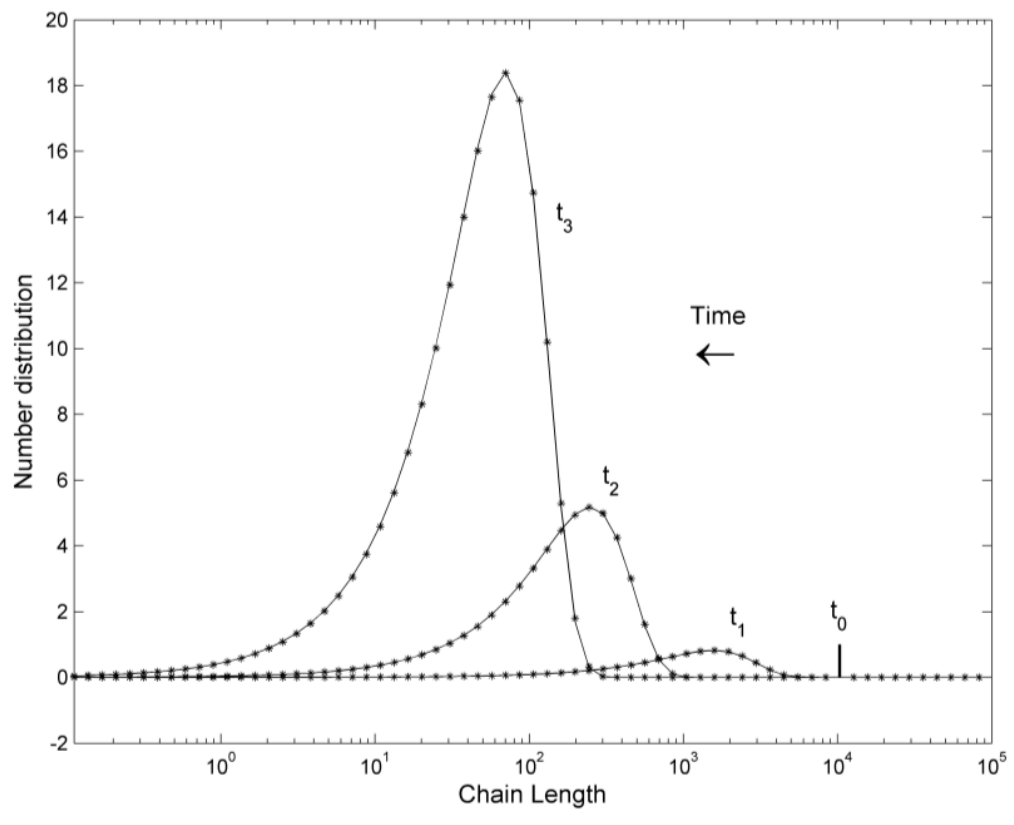

Figure 3a: Number distribution evolution: comparison between analytical (-) and numerical results $(*)$. The initial distribution is monodisperse and undertakes pure breakup with a breakage frequency $\Gamma=x$.

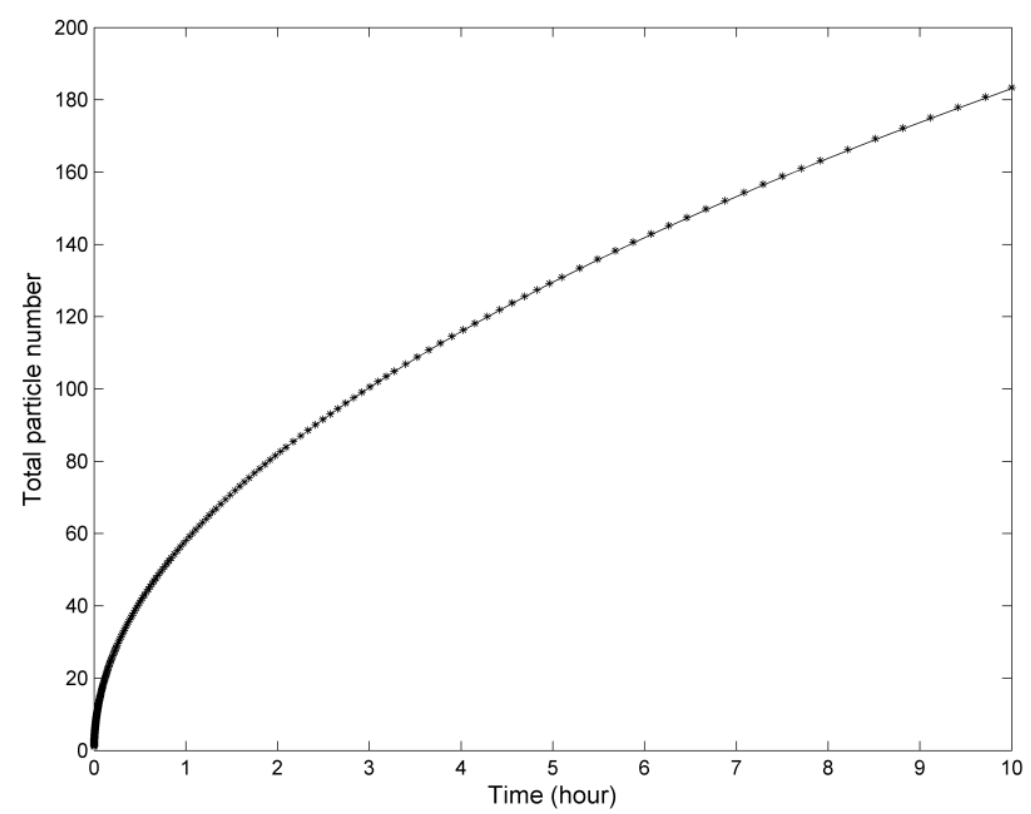

Figure 3b: Total particle number evolution under the endoglucanase activity, (-) analytical solution $(*)$ model simulation.

\section{Exoglucanase activity}

The initial pivots grid is defined according to an arithmetic sequence where the first pivot corresponding to the elementary particles produced by the exoglucanase chain-end-cleaving is 
fixed, when the others move at a constant rate. The cellobiose as the smallest and indestructible particle in such system is chosen for the fixed pivot.

As expected in the case of pure growth, the Figure 4a shows a constant translation without any deformation of the initial distribution. The number of chains represented by the pivots weight remains unchanged while all the cellobiose produced during the reaction accumulates on the first pivot as we can see in the Figure $4 \mathrm{~b}$. It can be noticed that the cellobiose production rate is constant until the left side of the distribution reaches the first pivot. From that point onward, the number of chains decreases meaning that the rate of cellobiose production becomes limited by the amount of available hydrolysable chains. At the end of the process, it can be seen that the mass conservation is satisfied since a $100 \%$ conversion is reached. This mass conservation after total hydrolysis validates the accuracy of the numerical procedure.

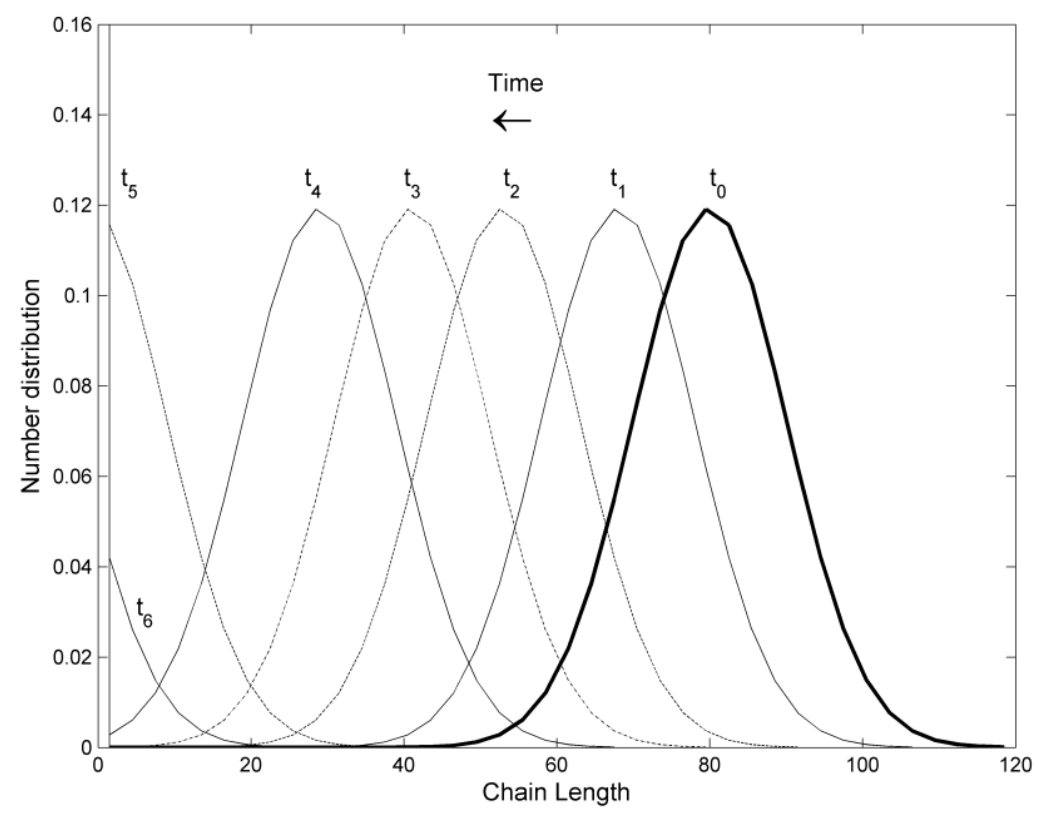

Figure 4a: Number distribution evolution under exoglucanase activity $(G=-5)$ 


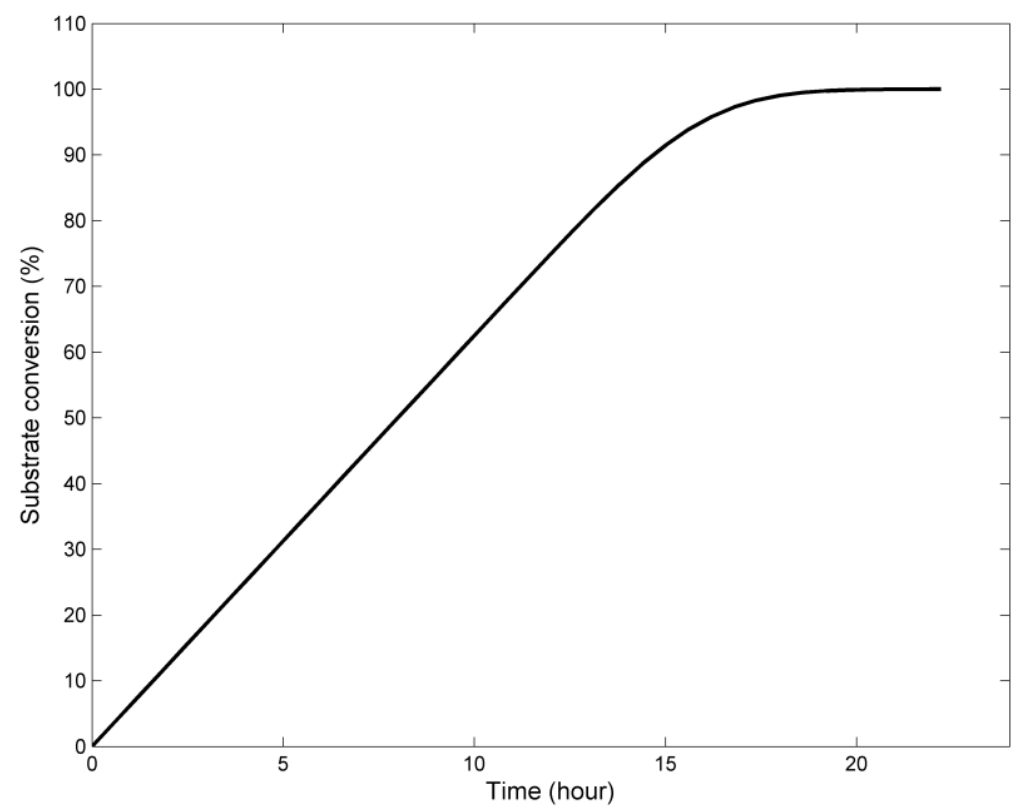

Figure 4b: Substrate conversion during hydrolysis by exoglucanases. The total mass is conserved

\section{Endoglucanase-Exoglucanase combination activity}

Once the models for the two main cellulolytic activities have been validated independently, they were combined in a global model accounting for both activities. The results obtained with a Gaussian initial size distribution are shown in the figures $5 \mathrm{a}$ and $5 \mathrm{~b}$.

As we can see in the Figure 5a, the combined endo/exoglucanase activity results in a progressive deformation of the initial number distribution. In fact, the particles size is reduced as a consequence of the exoglucanase action while new particles are produced by the chains cleavage leading to an increase in the number of small particles. Here again the mass balance is preserved along the conversion as illustrated in Figure 5b. In addition to that, if we assume that all the produced cellobiose is converted into glucose (disregarding the inhibition effect and the concentration of the $\beta$-glucosidases), the Figure $5 \mathrm{~b}$ is also representative of the cellulosic fraction converted into glucose. Knowing the initial amount of substrate, the produced glucose can be assessed at any given time. 


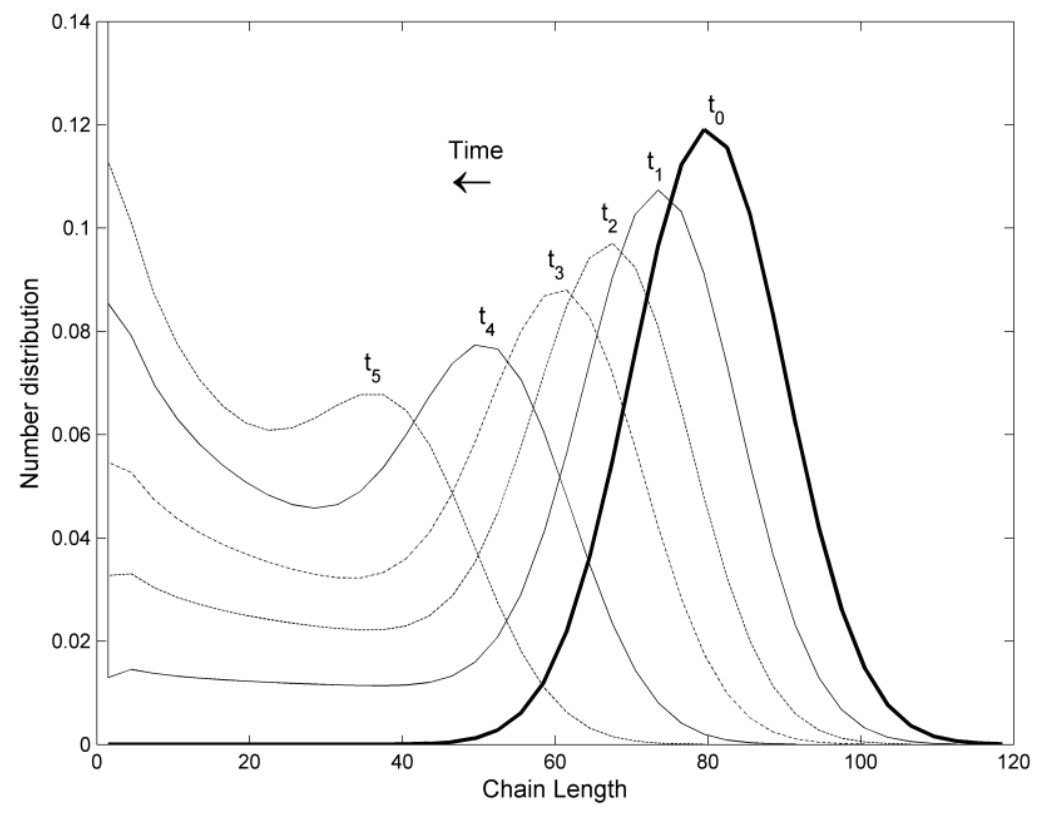

Figure 5a: Number distribution evolution under combined endo/exo activities $(\Gamma=0.25, G=-$ 10).

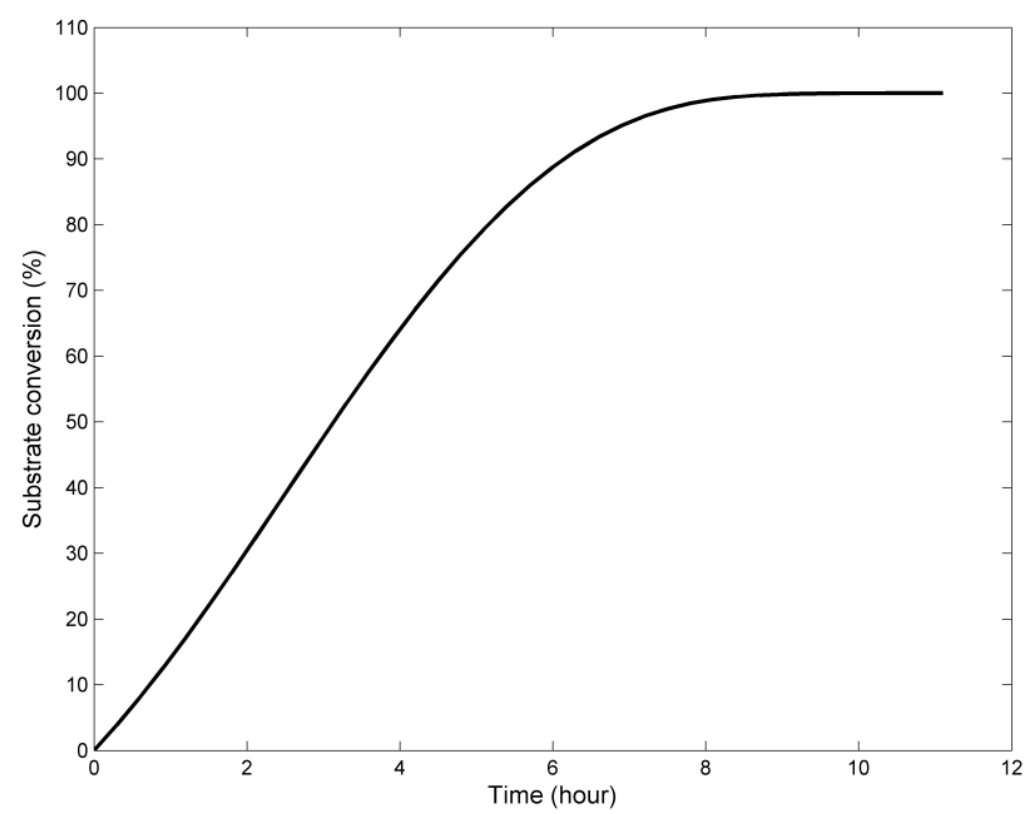

Figure 5b: Simulated substrate conversion during the hydrolysis considering endo and exo activities. The mass balance is conserved.

Generally, the elementary mechanisms of both cellulolytic activities can be recognized. In order to test the robustness of the model, we have compared the results obtained with various values for $\Gamma$ and $G$ which is analogous to testing different enzymes ratios (see the Table 1). In the Figure 6a, we report the results obtained when only the endoglucanase activity increases 
(which can be experimentally correlated with the enzyme concentration). In the Figure $6 \mathrm{~b}$, the endoglucanase activity remains constant while that of exoglucanase changes.

Table 1: Reaction time prediction (hours) for a conversion of $99 \%$ of the substrate under endo/exoglucanase activities.

\begin{tabular}{|c|c|c|c|c|}
\hline \multicolumn{1}{|r|}{$\Gamma$} & 0,01 & 0,5 & 1 & 2 \\
\hline 0,5 & 168 & 30 & 16 & 11 \\
\hline 5 & 18,4 & 12 & 8,4 & 5,6 \\
\hline 10 & 9,1 & 7,2 & 5,8 & 4,6 \\
\hline 15 & 6 & 5,2 & 4,5 & 3,5 \\
\hline
\end{tabular}

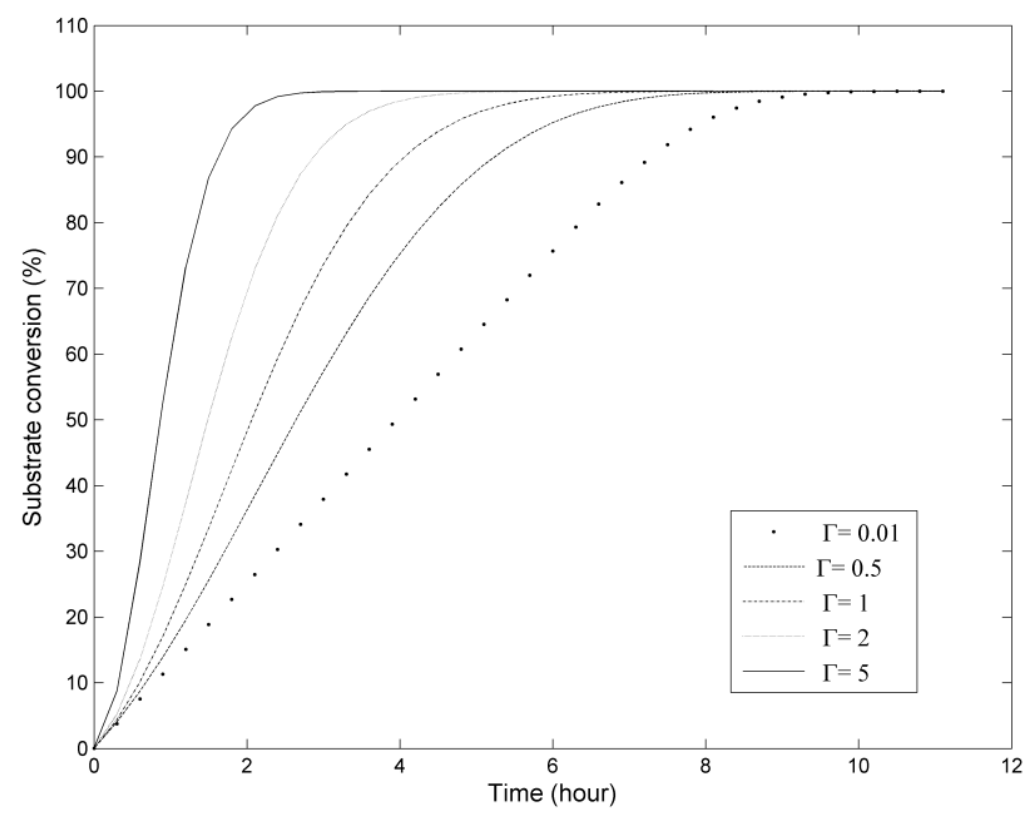

Figure 6a: Substrate conversion evolution under different endoglucanase rates $\Gamma$. 


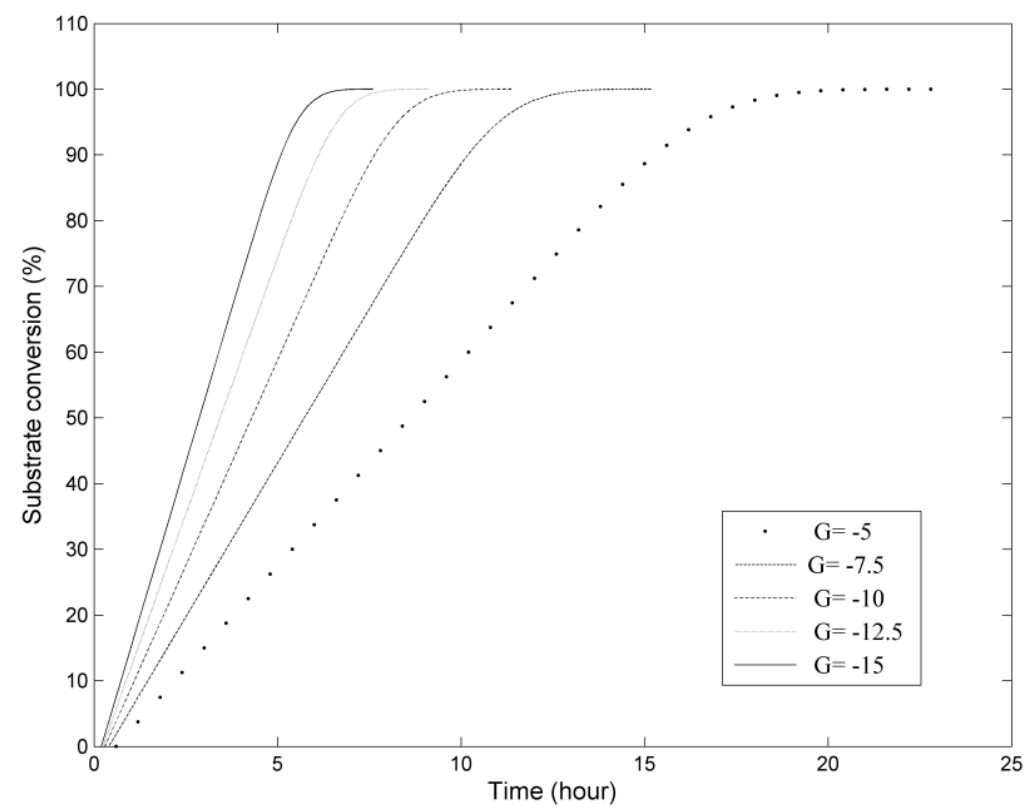

Figure 6b: Substrate conversion evolution under different exoglucanase rates $G$.

The increase on the activity of each enzyme leads to an enhancement of the cellulose hydrolysis rate, the maximum conversion is reached more rapidly. These results are consistent and show that the model offers an assessment of the contribution of each enzyme activity. Furthermore, the results obtained by the combined model have been compared to those given by the two models developed initialy for the two cellulolytic activities as shown by the Figure 7. The comparison between the sum of conversions obtained by the endoglucanase and the exoglucanase activities separately and that given by the combined model respecting the same rates for the two activities shows a difference due to the endo-exo synergism. These results confirm that the global model can predict and assess the synergy effect numerically according to the initialy imposed enzymes activity. 


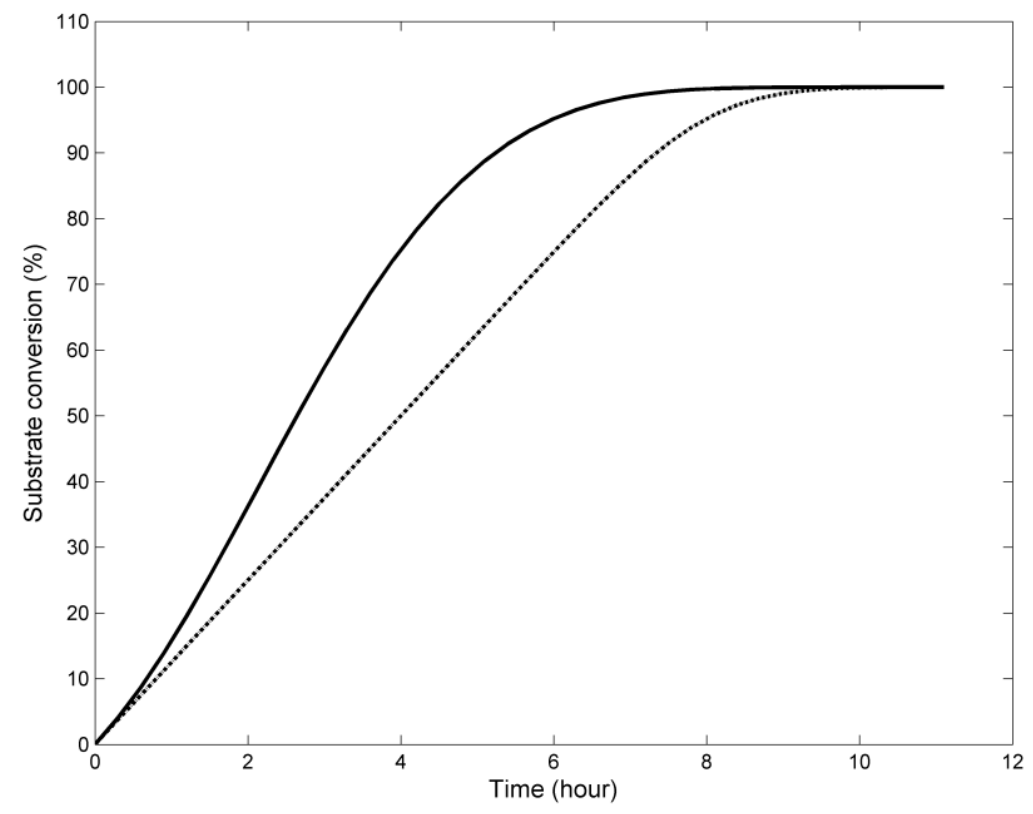

Figure 7: Substrate conversion during hydrolysis ( $\cdots$ ) endo+exo separately, (-) endo and exo in cocktail $(\Gamma=0.5, G=-10)$. The arrow quantifies the gain due to the synergistic effect of the two cellulases.

In order to test the applicability of the developed model to fit experimental data in term of fractional substrate conversion, we refer to the experimental work of Woodward et al. as an example. ${ }^{[34]}$ In their experiments, they have studied the enzymatic hydrolysis of microcrystalline cellulose (Avicel PH-105) using a mixture of endoglucanase/exoglucanase from Trichoderma reesei and $\beta$-glucosidase from Aspergillus niger. The initial average degree of polymerisation of Avicel PH-105 is $230 .^{[35]}$ We assume a normal initial size distribution of the polymers with a peak at 230 .

Model parameters (breakage frequency and moving rate) have been estimated assuming a maximal conversion of $40 \%$ of initial substrate mass. As shown in the Figure 8, the model prediction is very close to the experimental results. It must be highlighted that, due to the recalcitrant nature of microcrystalline cellulose (high crystallinity index) and other parameters affecting the reaction rate, incomplete conversion is observed. 


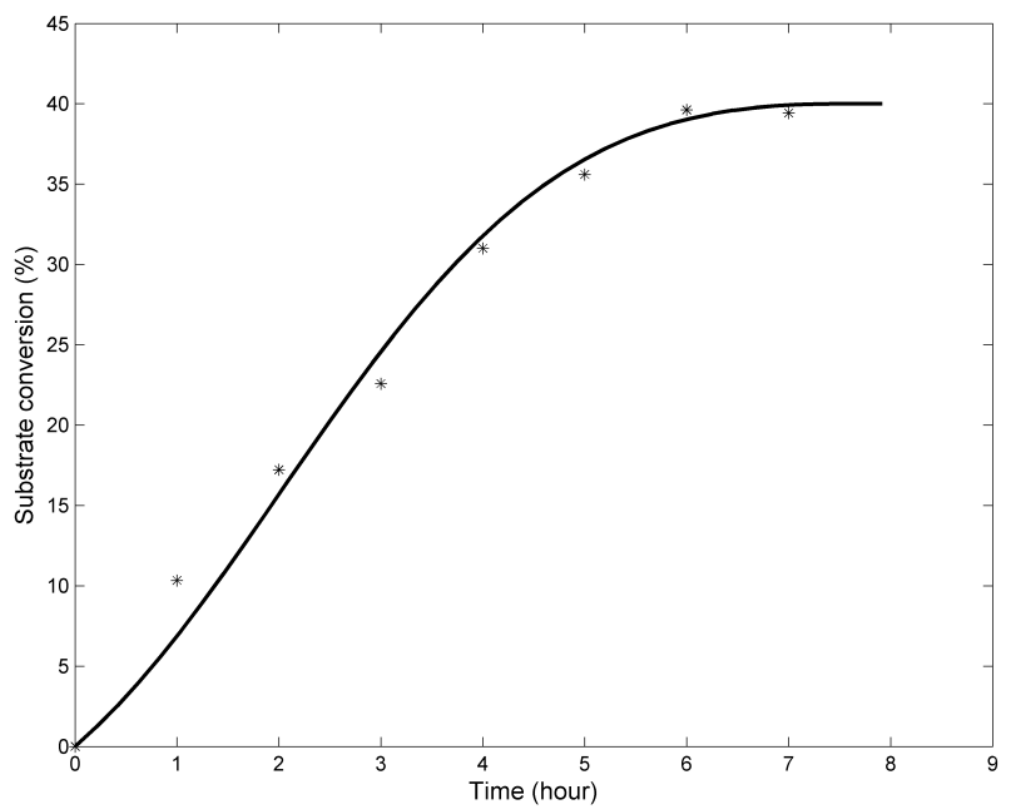

Figure 8: Substrate conversion of Avicel PH-105: (-) model prediction, (*) experimental results $^{[35]}(\Gamma=0.5, G=-48)$.

\section{Discussion}

The developed models for each of the two main cellulolytic activities show predictable results, successfully validated against analytical solutions. This section mainly focused on the results obtained when the two enzymatic activities are considered. Regarding the substrate conversion, typical enzymatic hydrolysis rate behavior characterized by a rapid initial rate and a progressive slowdown is reproduced by the model. In our case, the decrease of the overall hydrolysis rate is due to the substrate limitation (the number of hydrolysable chains finally goes to zero), and reaction stops when the converted fraction reaches $100 \%$.

As far as the number of chains is concerned, the total particles number increases because of the appearance of smaller chains in the system due to the endoglucanase activity that cleaves randomly the cellulose chains. The exoglucanase activity leads to a progressive dissolution of the chains. The associated particles mass loss produces cellobiose as a final product. The model gives access to the changes in the chains length distribution. The evolution of this distribution is markedly different depending on the magnitude of each enzymatic activity considered (Table 1). The increase in chain number is related to endoglucanases whereas the production of cellobiose is mostly due to the action of exoglucanases that leave the number of chains unchanged. 
It is remarkable that the substrate conversion curves (figures 6a and 6b) are very similar to that observed in most experiments. In that sense the numerical results could be fitted with a kinetic model (Michaelis-Menten model) to identify the apparent constants. However, we can claim that the apparent values of the parameters $V_{\max }$ and $K_{m}$ would be dependent on the actual values of $\Gamma$ and $G$. It is significant that the Michaelis-Menten like shape of the conversion curve does not come from the combination of multiple enzymatic reaction models: $\Gamma$ and $G$ are true constants.

The numerical parameters $\Gamma$ and $G$ are considered as constants in this first step of the model development. It is proposed here to relate them to the enzymatic activities. On one hand, the break-up frequency $(\Gamma)$ expressed as the number of chains breakages by time unit is easily turned into an enzymatic activity as defined in literature by a simple units conversion (breakages number to molar amount of reduced sugars).

$$
U=\frac{\Gamma}{\mathscr{N}}
$$

Where $T$ is the Avogadro number and $U$ the activity in mole of product (reduced sugars in our case) formed by unit time.

On the other hand, the amount of produced cellobiose can be deduced from the constant rate $(G)$ used for modeling the exoglucanase activity, as follows.

During the hydrolysis by exoglucanases, the number of the chains is constant. Assuming that the chains are polymers of a known monomer (ex. glucose) with an initial total mass $m_{T}$ and that the degree of polymerization $(D P)$ of the substrate is known, the initial number of chains is given by:

$$
N(0)=\frac{m_{T}}{M_{G l u} D P}
$$

If one assumes that the amount of enzyme is sufficient to attack all chains, the amount of cellobiose formed by time unit due to the action of exoglucanases is:

$$
\frac{d m_{c}}{d t}=\frac{G}{2} \frac{N(0)}{/} M_{\text {Cello }}
$$


Recall that $G=d x / d t$, that is to say the rate of chain length reduction expressed in monomers. The coefficient 2 indicates that two monomers are needed to form one cellobiose molecule. Then $G / 2$ times $N(0)$ represents the number of molecules of cellobiose formed by unit time.

$$
U=\frac{1}{M_{\text {Cello }}} \frac{d m_{c}}{d t}=\frac{G}{2} \frac{N(0)}{\mathscr{C}}
$$

Knowing the cellobiose releasing rate, the exoglucanase activity $(U)$ can be easily deduced.

When the three major hydrolytic enzyme activities (endoglucanase, exoglucanase and $\beta$ glucosidase) are combined, it is demonstrated (see Figure 7) that the hydrolysis rate exceeds the sum of the rates of the single enzymes showing a synergistic interaction of the cellulases system. ${ }^{[36]}$ Different synergisms depending on the nature of the cellulase system have been reported, ${ }^{[9,38]}$ the main one is between endoglucanase and exoglucanase called endo-exo synergism. ${ }^{[10,39]}$ The endoglucanase internal chain-cleaving makes available additional chain ends for the action of exoglucanases, that's why, when the reaction is not limited by the concentration of exoglucanases, the hydrolysis rate increases.

One of the critically important barriers affecting the enzymatic hydrolysis rate is the endproducts inhibition. The accumulation of cellobiose and glucose is partially responsible for the deceleration of the cellulases activity even at low degree of conversion of the substrate. This means that the effectiveness of the hydrolytic enzymes falls as the end-products concentration increases in the reactor. Incorporating this phenomenon in the population balance model is quite straightforward. The model parameters characterizing the two main activities (breakage frequency and growth rate) have to be related instantaneously to the endproducts concentration. We give in the equations (11) and (12) the kinetic rate expressions used in the case of non-competitive inhibition. The degree of inhibition in the system is controlled numerically by the constants $K_{\Gamma}$ and $K_{G}$. The specific constants $\mathrm{K}_{\Gamma}$ and $\mathrm{K}_{\mathrm{G}}$ can be experimentally determined. Here, they are set to arbitrary values. $\Gamma$ and $G$ are no longer constants, they are inversely proportional to the end-products concentration.

$$
\begin{aligned}
\Gamma\left(C_{s}\right) & =\Gamma_{0} \frac{K_{\Gamma}}{C_{S}+K_{\Gamma}} \\
G\left(C_{s}\right) & =G_{0} \frac{K_{G}}{C_{S}+K_{G}}
\end{aligned}
$$


In the Figure 9, we compare the degree of conversion of the same initial chains population with and without taking into account the inhibition effect. These numerical results are obtained by the population balance model.

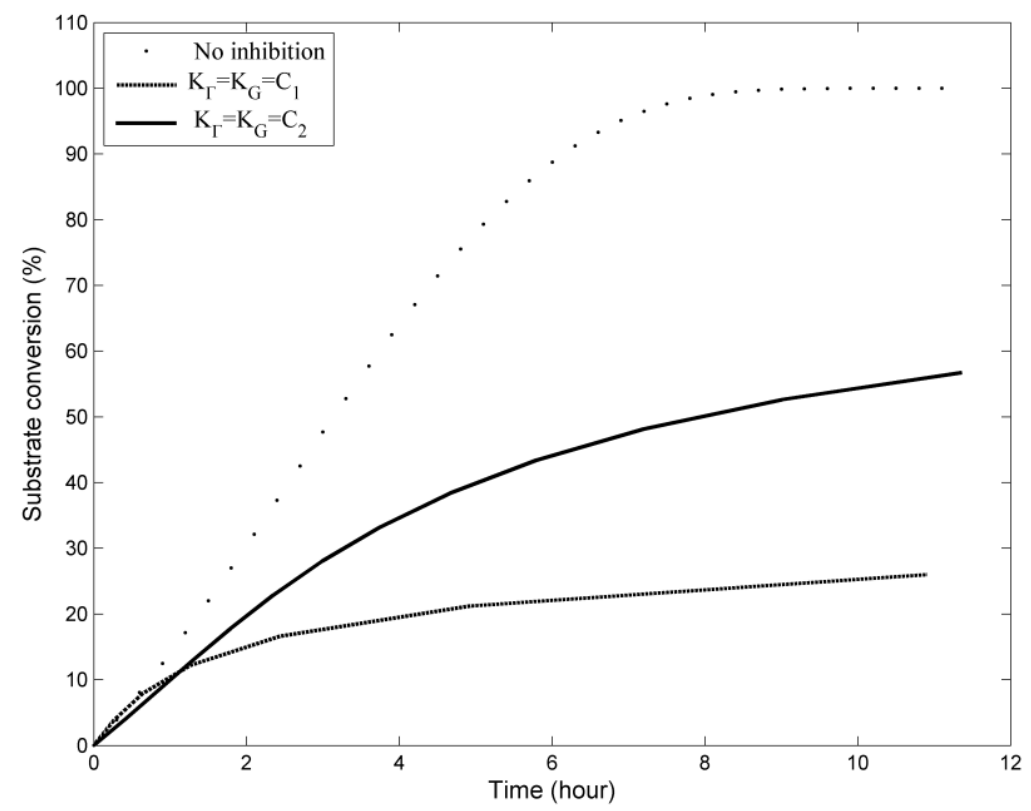

Figure 9: The inhibition effect. The cellulases activities slow down with conversion leading to a partial substrate conversion. Two cases with different inhibition constants are shown $(\Gamma(0)=0.25, G(0)=-10)$.

The implementation of the new parameters expressions in the population balance model gives interesting results. Unlike the previous cases where the enzymes activities are only limited by the availability of the substrate leading finally to a total conversion, the introduction of the inhibition effect reflects more the reality, the substrate conversion rate falls drastically thus, the substrate is partially converted.

The results obtained in this work in terms of the substrate particles size distribution evolution during enzymatic hydrolysis are qualitatively similar to those obtained experimentally by Mooney et al. ${ }^{[39]}$ who measured the fiber length distribution of Douglas-fir kraft pulp during enzymatic hydrolysis (figure 10). A direct comparison between our model and these data was not attempted since the data correspond to fibers. Indeed, in the latter case, one has to think about how to express the effectiveness of each enzyme activity knowing that during the reaction, the two soluble/insoluble substrate systems coexist. Nevertheless, the similarity is encouraging and this tends to demonstrate that the numerical approach proposed here can be generalized to insoluble particles. 


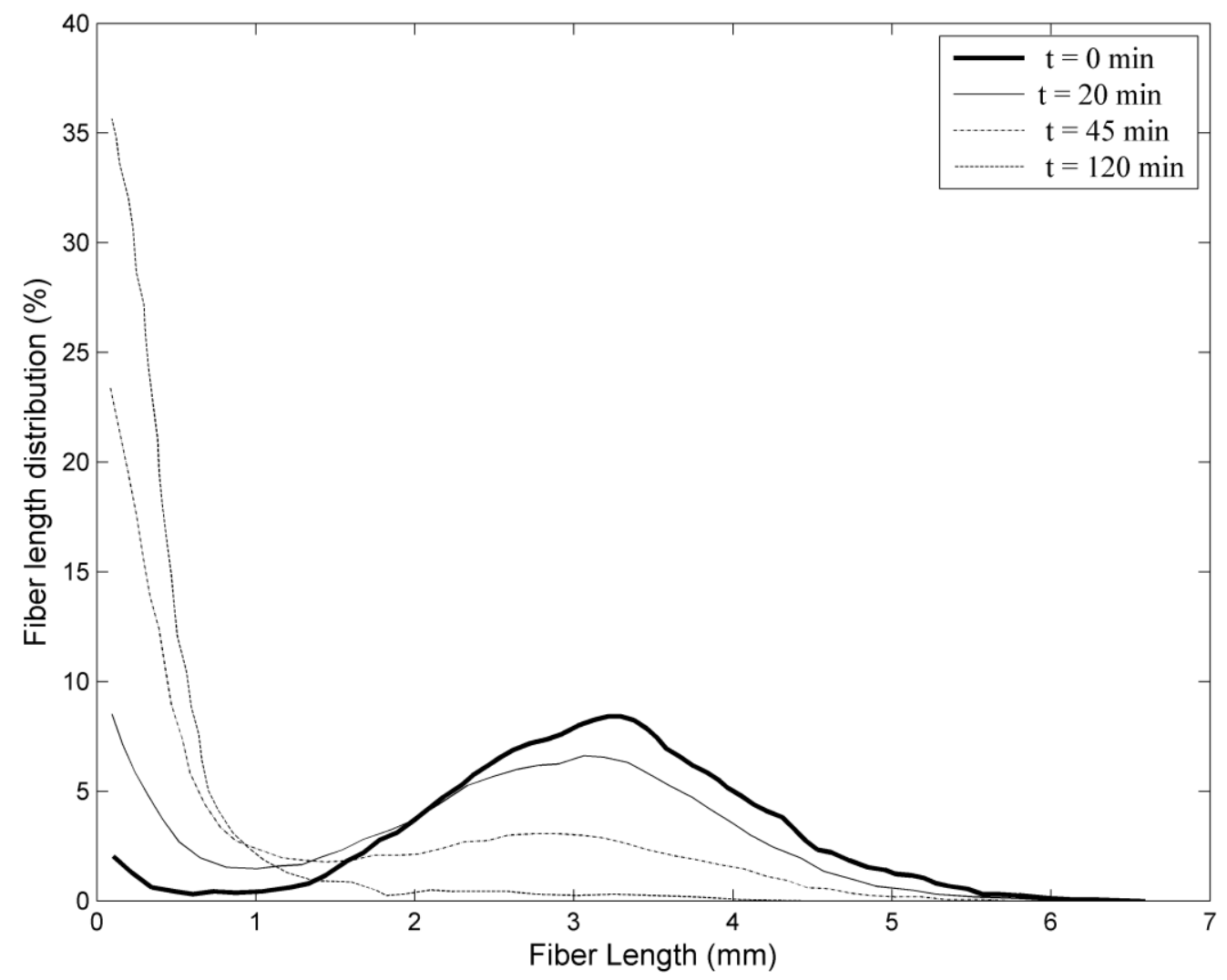

Figure 10: Changes in population fiber-length distribution of Douglas fir kraft pulp resulting from hydrolysis ${ }^{[40]}$.

If one assumes that the $\beta$-glucosidase activity is such that the conversion of cellobiose into glucose is instantaneous, then the glucose concentration should evolve as the cellobiose concentration. But if one considers also the inhibitory effect on $\beta$-glucosidase, the whole degradation kinetic will become dependent on the three enzymatic activities. These inhibitory effects have been identified ${ }^{[40]}$ and can be further incorporated in the model through the introduction of a kinetic rate expression for the $\beta$-glucosidase.

Experimental data, ${ }^{[34]}$ as those of major works, ${ }^{[42,43]}$ show partial substrate conversion because of its decreasing reactivity due to several parameters: changes in cristallinity index, ${ }^{[43]}$ accessible surface area ${ }^{[8]}$ and chemical composition. ${ }^{[44]}$ Incorporating these factors and their interactive effects in the model requires the development of the appropriate parameters expressions (this is done in the case of end-products inhibition effect) or the addition of other dimensions to the existing model. 


\section{Conclusion}

A population balance based kinetic model has been developed for the enzymatic hydrolysis of cellulosic materials. The pure cellulose has been assimilated to polymer chains with given initial length distribution leading, in the case of enzymatic hydrolysis, to a depolymerisation process. Firstly, the two main activities have been modeled separately in order to validate the numerical approach. A discretization method has been used for the resolution with a fixed pivot technique for the endoglucanase activity and a moving pivot technique for the exoglucanase action. Afterwards, a combined model for endoglucanase-exoglucanase activity has been proposed. Under some assumption, the kinetics of final product release are also readily accessible through this model. This model has been tested for different enzyme ratios and shows coherent results. In addition, the comparison between the conversion obtained by the global model and those given by the two initial models separately in the same conditions shows a difference attributed to the endo-exo synergism. Since the enzymatic activity is inhibited by the products, this critically important phenomenon is incorporated in the model. Finally, the prediction of the population balance model compares favorably with the experimental results available in the literature.

In this study, the formulation of a one-dimensional model is based only on the hydrolytic enzyme elementary mechanisms known in the literature disregarding other factors affecting cellulose hydrolysis rate. The principal aim was the validation of the population balance approach using simple mathematical breakage frequency and growth rate expressions. According to the results, this approach presents a real potential for a better description of the enzymatic hydrolysis of such complex systems.

\section{Nomenclature}

$\begin{array}{ll}x, x & \text { Chain length } \\ t & \text { Time } \\ n(x, t) & \text { Number density of chains with a length } x \text { at time } t \\ G(x) & \left.\text { Growth rate for chains of length } x \text { [monomer. } \mathrm{t}^{-1}\right] \\ \Gamma(x) & \text { Breakage frequency for a chain of length } x\left[\mathrm{t}^{-1}\right]\end{array}$




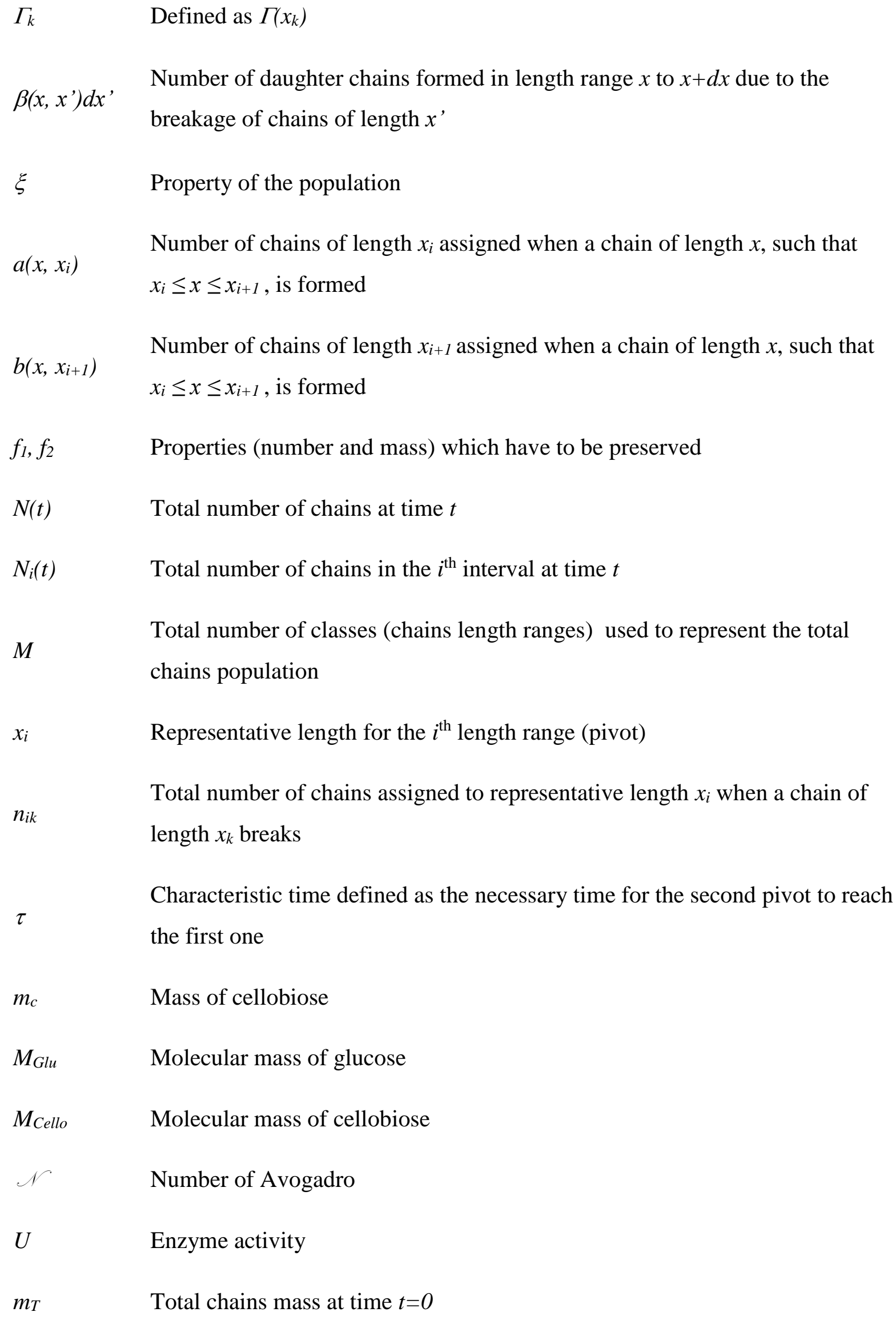


$K_{\Gamma}, K_{G} \quad$ Constants introduced for the non-competitive inhibition

$C_{S} \quad$ End-products concentration

\section{References}

[1] H. Jørgensen, J.B. Kristensen, C. Felby, Biofuels Bioprod. Biorefining 2007, 1, 119.

[2] G.P. Philippidis, C.E. Wyman, Recent Adv. Biotechnol. 1992, 405.

[3] Y. Sun, J. Cheng, Bioresour. Technol. 2002, 83, 1.

[4] J.S. Van Dyk, B.I. Pletschke, Biotechnol. Adv. 2012, 30, 1458.

[5] J.B. Kristensen, Enzymatic Hydrolysis of Lignocellulose. Substrate Interactions and High, 2008.

[6] M. Chauve, H. Mathis, D. Huc, D. Casanave, F. Monot, N.L. Ferreira, Biotechnol. Biofuels 2010, 3, 3 .

[7] L. Lin, R. Yan, Y. Liu, W. Jiang, Bioresour. Technol. 2010, 101, 8217.

[8] P. Bansal, B.J. Vowell, M. Hall, M.J. Realff, J.H. Lee, A.S. Bommarius, Bioresour. Technol. 2012, 107, 243.

[9] J. Medve, J. Karlsson, D. Lee, F. Tjerneld, Biotechnol. Bioeng. 1998, 59, 621.

[10] E. Hoshino, M. Shiroishi, Y. Amano, M. Nomura, T. Kanda, J. Ferment. Bioeng. 1997, $84,300$.

[11] J.R. Samaniuk, C. Tim Scott, T.W. Root, D.J. Klingenberg, Bioresour. Technol. 2011, 102, 4489.

[12] Y. Chen, Y. Wen, J. Zhou, C. Xu, Q. Zhou, Bioresour. Technol. 2012, 110, 321.

[13] S.E. Levine, J.M. Fox, H.W. Blanch, D.S. Clark, Biotechnol. Bioeng. 2010, 107, 37.

[14] P. Bansal, M. Hall, M.J. Realff, J.H. Lee, A.S. Bommarius, Biotechnol. Adv. 2009, 27, 833.

[15] R.M.F. Bezerra, A.A. Dias, Appl. Biochem. Biotechnol. 2004, 112, 173.

[16] Y.P. Teoh, M. Mat Don, Chem. Eng. Sci. 2011, 66, 3523.

[17] Q. Gan, S.. Allen, G. Taylor, Process Biochem. 2003, 38, 1003.

[18] Y.-H.P. Zhang, L.R. Lynd, Biotechnol. Bioeng. 2006, 94, 888.

[19] A.O. Converse, H. Ooshima, D.S. Burns, Appl. Biochem. Biotechnol. 1990, 24-25, 67.

[20] D. Lee, A.H.C. Yu, K.K.Y. Wong, J.N. Saddler, Appl. Biochem. Biotechnol. 1994, 45$46,407$.

[21] K. Ohmine, H. Ooshima, Y. Harano, Biotechnol. Bioeng. 1983, 25, 2041.

[22] A.P. Sinitsyn, O.V. Mitkevich, A.V. Gusakov, A.A. Klyosov, Carbohydr. Polym. 1989, $10,1$.

[23] J. Hong, X. Ye, Y.-H.P. Zhang, Langmuir 2007, 23, 12535.

[24] M. Okazaki, M. Moo-Young, Biotechnol. Bioeng. 1978, 20, 637.

[25] K. Movagarnejad, M. Sohrabi, T. Kaghazchi, F. Vahabzadeh, Biochem. Eng. J. 2000, 4, 197.

[26] S.A. Hosseini, N. Shah, Biomass Bioenergy 2011, 35, 3841.

[27] S.A. Hosseini, N. Shah, Biomass Bioenergy 2011, 35, 3830.

[28] S. Kumar, D. Ramkrishna, Chem. Eng. Sci. 1996, 51, 1311.

[29] S. Kumar, D. Ramkrishna, Chem. Eng. Sci. 1996, 51, 1333.

[30] S. Kumar, D. Ramkrishna, Chem. Eng. Sci. 1997, 52, 4659.

[31] R.M. Ziff, E.D. McGrady, J. Phys. Math. Gen. 1999, $18,3027$.

[32] B.J. McCoy, G. Madras, Chem. Eng. Sci. 2001, 56, 2831. 
[33] G. Madras, B.J. McCoy, AIChE J. 2004, 44, 647.

[34] J. Woodward, M.K. Hayes, N.E. Lee, Nat. Biotechnol. 1988, 6, 301.

[35] M. Jumaa, F.E. Saleh, I. Hassan, B.W. Müller, P. Kleinebudde, Colloid Polym. Sci. 2000, 278, 597.

[36] B. Nidetzky, W. Steiner, M. Hayn, M. Claeyssens, Biochem. J. 1994, 298, 705.

[37] D.W. Kim, Y.G. Hong, Biotechnol. Bioprocess Eng. 2001, 6, 89.

[38] Y.-H.P. Zhang, L.R. Lynd, Biotechnol. Bioeng. 2004, 88, 797.

[39] C.A. Mooney, S.D. Mansfield, R.P. Beatson, J.N. Saddler, Enzyme Microb. Technol. 1999, 25, 644.

[40] T. Eriksson, J. Karlsson, F. Tjerneld, Appl. Biochem. Biotechnol. 2002, 101, 41.

[41] L. Zhu, J.P. O’Dwyer, V.S. Chang, C.B. Granda, M.T. Holtzapple, Bioresour. Technol. 2008, 99, 3817.

[42] B.T. Smith, J.S. Knutsen, R.H. Davis, Appl. Biochem. Biotechnol. 2010, 161, 468.

[43] M. Hall, P. Bansal, J.H. Lee, M.J. Realff, A.S. Bommarius, FEBS J. 2010, 277, 1571.

[44] X. Zhao, L. Zhang, D. Liu, Biofuels Bioprod. Biorefining 2012, 6, 465. 